\title{
A Decade of Coronagraphic and Spectroscopic Studies of CME-Driven Shocks
}

\author{
Angelos Vourlidas* and Alessandro Bemporad ${ }^{\dagger}$ \\ * Space Sciences Division, Naval Research Laboratory, Washington DC, USA \\ ${ }^{\dagger}$ INAF-Osservatorio Astronomico di Torino, Pino Torinese, Italy
}

\begin{abstract}
Shocks driven by Coronal Mass Ejections (CMEs) are primary agents of space weather. They can accelerate particles to high energies and can compress the magnetosphere thus setting in motion geomagnetic storms. For many years, these shocks were studied only in-situ when they crossed over spacecraft or remotely through their radio emission spectra. Neither of these two methods provides information on the spatial structure of the shock nor on its relationship to its driver, the CME. In the last decade, we have been able to not only image shocks with coronagraphs but also measure their properties remotely through the use of spectroscopic and image analysis methods. Thanks to instrumentation on STEREO and $S O H O$ we can now image shocks (and waves) from the low corona, through the inner heliosphere, to Earth. Here, we review the progress made in imaging and analyzing CME-driven shocks and show that joint coronagraphic and spectrscopic observations are our best means to understand shock physics close to the Sun.
\end{abstract}

Keywords: Coronal Mass Ejections, Interplanetary shocks, Shocks in Plasma,Coronagraphs PACS: $96.60 . \mathrm{Ph}, 96.50 . \mathrm{Fm}$, 52.35.Tc, $95.55 . \mathrm{Fw}$

\section{THE STUDY OF CME-DRIVEN SHOCKS NEAR THE SUN}

Space-based coronagraphs have observed thousands of Coronal Mass Ejections (CMEs) since their discovery in the early 1970s. The observations show that hundreds of CMEs can attain speeds of $1000 \mathrm{~km} / \mathrm{sec}$ or more within a few $\mathrm{R}_{\odot}$ which exceed the local Alfvén speed at those heights. Hence, fast CMEs can drive shocks which are detected sometimes in the radio wavelengths as drifting type-II bursts [6]. Such indirect observations, however, provide only limited information about the shock; namely, the driver speed and, the onset and duration of the radio emission.

Fortunately, direct imaging and spectroscopic measurements of the density compression at the shock front have been made possible in the last ten years thanks to the LASCO coronagraphs and UVCS spectrometer, both instruments aboard the Solar Heliospheric Observatory $(\mathrm{SOHO})$. As we will explain, the combination of white light and UV observations can provide the full range of physical parameters (density, temperature, magnetic field) across the shock in addition to spatial information about the shock shape and its relation to the driving CME. Now, the physics of coronal shocks can be studied in detail. The off-limb spectroscopy and coronagraphy are, and will be, our only means for studying shocks where they can accelerate particles with the highest energies $\left(<10 \mathrm{R}_{\odot}\right)$. Even the forthcoming Solar Probe Plus mission will only reach to $9.5 \mathrm{R}_{\odot}$. 

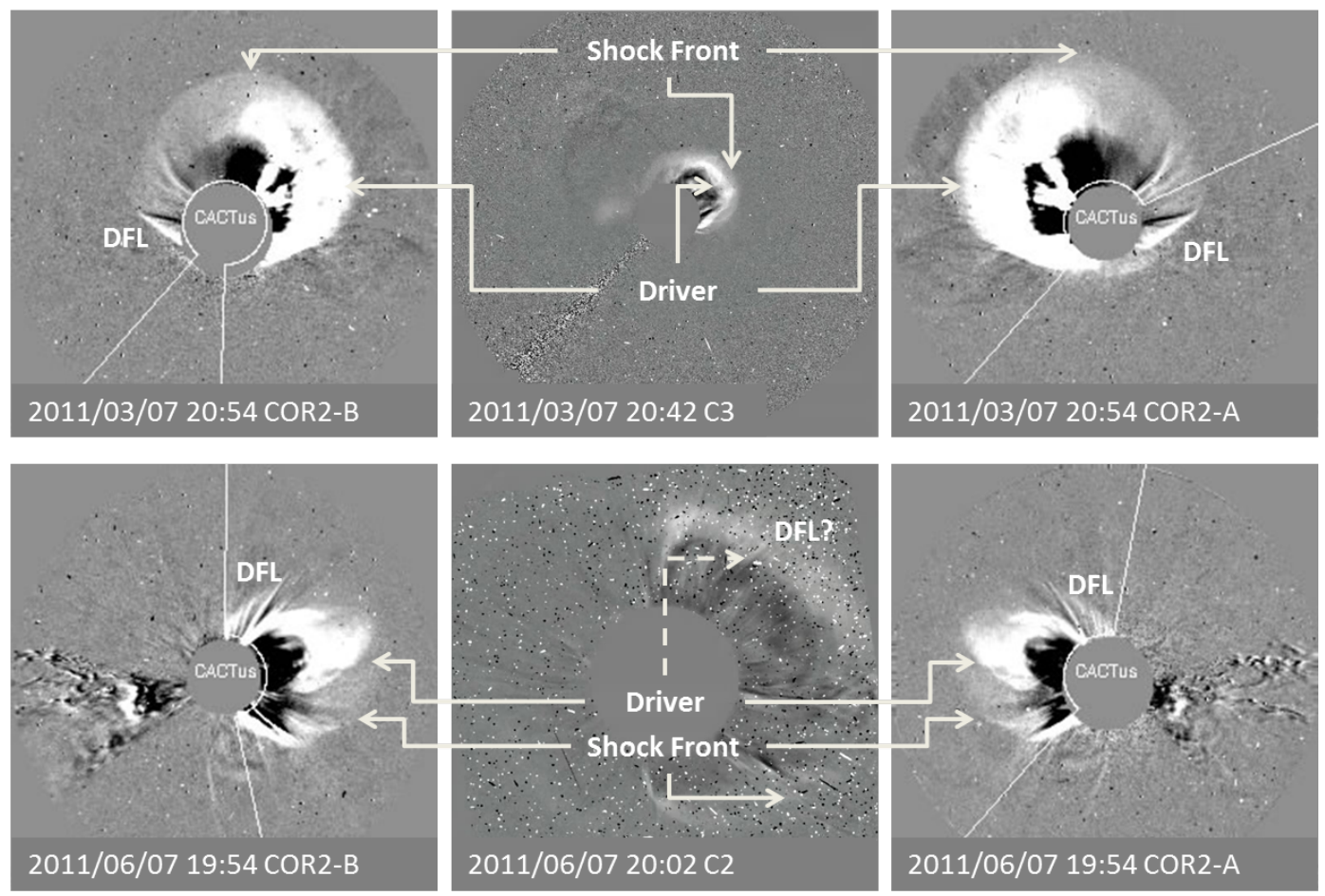

FIGURE 1. Multipoint coronagraph observations of CME-driven shocks. The times and telescopes are shown on the figure. The angular distance from Earth for COR2-A (COR2-B) are $88^{\circ}\left(95^{\circ}\right)$ on $03 / 07$ and $95^{\circ}\left(93^{\circ}\right)$ on $06 / 07$, respectively. Several streamers are clearly deflected by the shock. Some examples are denoted with 'DFL'. For the June $6 \mathrm{CME}$, the driver cannot be readily distinguished from the deflected streamers.

\section{CORONAGRAPHIC IMAGING OF SHOCKS}

Because we have reviewed many aspects of coronagraphic imaging of CME shocks in a recent volume of this conference series [25], we present here only a brief overview of the progress made over the last two years. The early work on shock detection $[24,18,25]$ has motivated an ever expanding number of detections of CME-driven shocks [7, 22, 21, 16$]$. These works are based mostly on observations by the SECCHI instruments which have higher sensitivites than the LASCO coronagraphs. The white light shocks can now be routinely detected in the inner corona, $\sim 1.55 \mathrm{R}_{\odot}$ [7], and can be followed out to at least $0.5 \mathrm{AU}[16]$. The SECCHI and radio observations show that shocks tend to form at 1.5 $\mathrm{R}_{\odot}$ and can accelerate electrons out to $4 R_{\odot}$ [7]. The standoff distance between the shock and the driver appears to be $1-2 \mathrm{R}_{\odot}$ within the coronagraph field of view $[7,16,8]$. Clear white light shock signatures were rare during 2007-2010 due to the prolonged activity minimum but there have been several fast events since February 2011. We present two recent examples in Figs. 1-2. The three viewpoints afforded by the SECCHI and LASCO telescopes easily reveal the global reach of CME-driven shocks in the middle corona. 

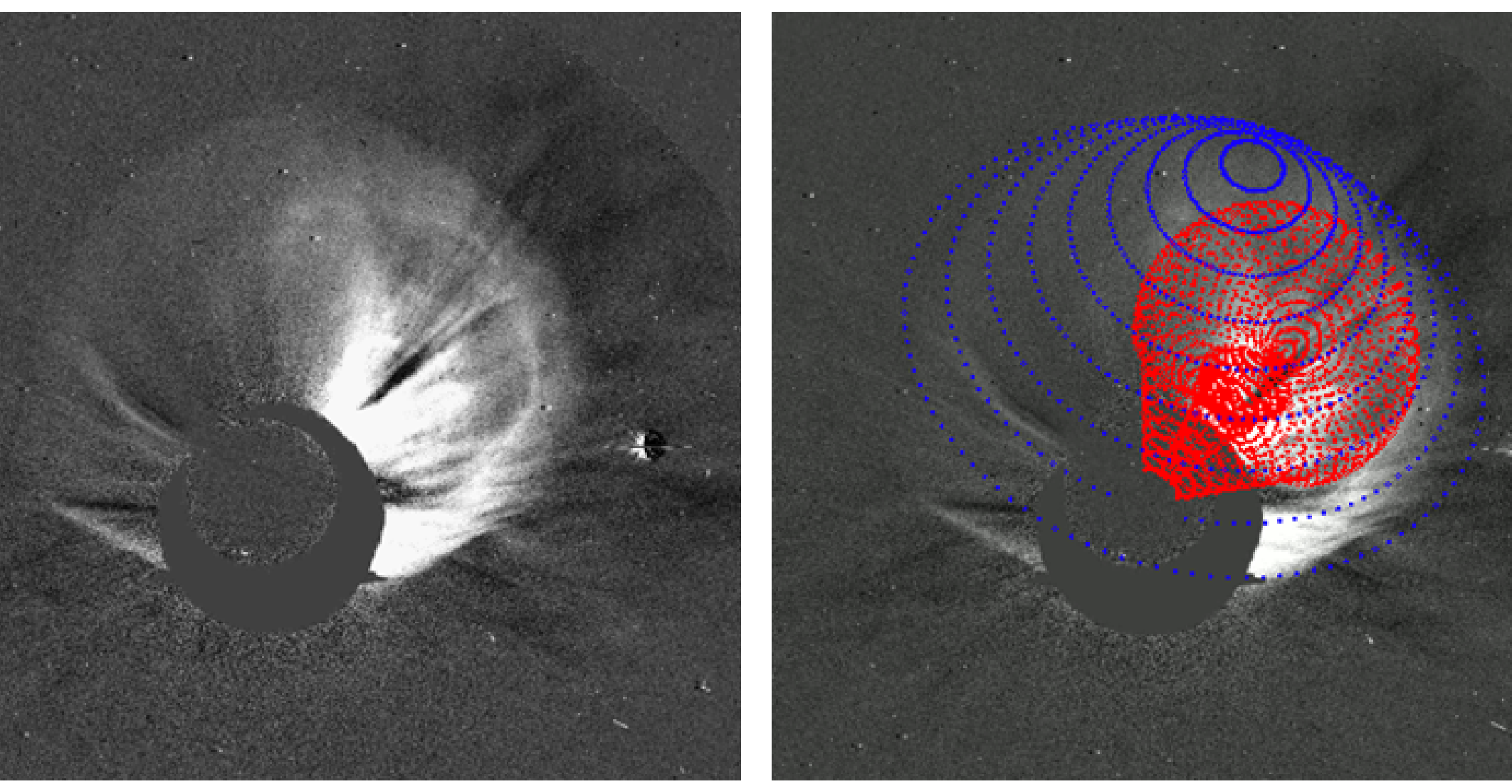

FIGURE 2. Example of a 3D fitting of the shock and its CME driver for the event on March 7, 2011 using the forward modeling method in $[18,23]$.

Measurements of the shock longitudinal extent allow us to localize with better precision the injection of Solar Energetic Particles (SEPs) along magnetic field lines [21] and connect it to the CME source region [22].

\section{SPECTROSCOPIC MEASUREMENTS OF SHOCKS}

Shocks can be identified in UV spectra by means of heating, density compression and high speeds associated with them. The first spectral detection of a CME driven shock was made by UVCS in 2000 [20]. The authors measured enhancements in the $\mathrm{O}^{5+}$ and $\mathrm{Si}^{11+}$ that were consistent with an MHD shock. More than a dozen of shocks have been identified since $[12,3,4,13,14,2,15]$. A review of plasma physical parameters derived by UVCS across shocks is given in Table 1 . The plasma heating and compression across the shock lead typically to strong brightening of the $\mathrm{Si}^{11+}$ (Si XII $\lambda 521$ ) line, brightening or dimming of the $\mathrm{O}^{5+}(\mathrm{O}$ VI $\lambda \lambda 1032-1037)$ lines and dimming of the H I Ly $\alpha \lambda 1216$ line. These differences are related to the different physical processes involved in the atomic excitations, because coronal ions are mainly excited by both collisions with electrons (collisional excitation) and/or by resonant scattering of photons emitted from the underlying layers of the solar atmosphere (radiative excitation); the latter process is 

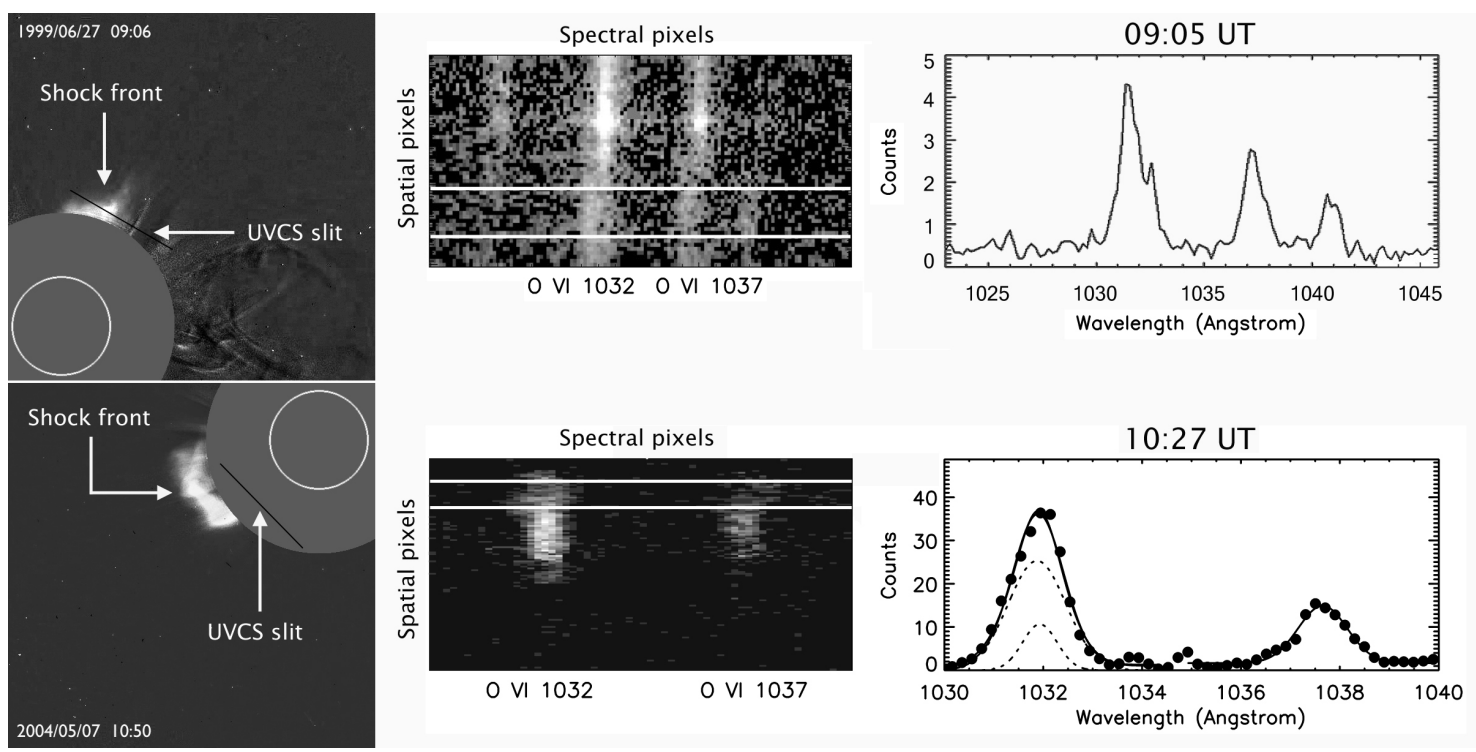

FIGURE 3. Two examples of shock detections by UVCS. Top left: LASCO/C2 running difference image showing the June 27, 1999 event and the location of the UVCS slit (solid black line) during the observations. Top middle: $\mathrm{O}$ VI $\lambda \lambda$ 1032-1037 intensities observed during the transit of the CME-driven shock. Top right: line profile broadenings observed at the shock transit [average over the spatial region shown in the middle panel by the two solid white lines; 19]. Bottom: same as top panels, but for the May 7, 2004 event [15].

also related to the Doppler dimming effect [see 17].

The most evident signature of a shock in EUV spectra is a broadening of the $\mathrm{O} V \mathrm{VI}$ $\lambda \lambda$ 1032-1037 line profiles in close correspondence to the shock transit, as derived from shock speeds either measured in the LASCO coronagraph images or derived from the frequency slope of accompanying type-II radio bursts (Fig. 3). Spectral signatures of the shocks have been detected from about $1.7 \mathrm{R}_{\odot}$ up to $4.3 \mathrm{R}_{\odot}$ (Table 1 ). The observed broadening is usually reproduced with superposition of a narrower component due to emissison from coronal plasma located along the line of sight (LOS), and a broader component due to the shock emission (e.g., Fig. 3 bottom right panel). The latter is often red- or blue-Doppler shifted by up to $0.4 \AA$ with respect to the coronal emission because of the LOS component of shock velocity, resulting in asymmetric observed profiles [see e.g., 19, 2, and Fig. 3, top right panel]. FWHMs of broad components typically correspond to post-shock $\mathrm{O}^{5+}$ kinetic temperatures $T_{k} \sim 10^{8} \mathrm{~K}$. Broadenings observed in spectral lines emitted by different ions/elements are representative of heatings occurring for different charge-to-mass ratios, thus providing important information on physical mechanisms occurring across the shock surface. For instance, [12] pointed out that the observed O VI $\lambda 1032$ line broadening can be explained by the mechanism proposed by [11], where the heating at a quasi-perpendicular shock is due to the nondeflection of ions at the shock ramp. For another event, [13] concluded that observed deficiency of $\mathrm{O}^{5+}$ ion heating might be attributable to different local plasma conditions, such as higher values of the $\beta$ parameter (i.e. thermal over magnetic plasma pressure) in the corona crossed by the shock. This would be in agreement with [10], who found that, for supercritical 
TABLE 1. CME-driven Shock Parameters Derived from UVCS data

\begin{tabular}{ccrrrrr}
\hline \multicolumn{1}{c}{ Date } & $\begin{array}{c}\text { Height } \\
\left(\mathbf{R}_{\odot}\right)\end{array}$ & $\begin{array}{c}\text { Speed } \\
\left(\mathbf{k m ~ s}^{-1}\right)\end{array}$ & $\begin{array}{c}\text { Density } \\
\left(10^{6} \mathbf{c m}^{-3}\right)\end{array}$ & $\mathbf{L o g}\left(\mathbf{T}_{k}\right)$ & $\mathbf{X}$ & Reference \\
\hline $06 / 11 / 98$ & 1.75 & 1200 & 1 & 8.7 & 1.8 & {$[20]$} \\
$06 / 27 / 99$ & 2.55 & 1200 & - & $<8.2$ & - & {$[19]$} \\
$03 / 03 / 00$ & 1.70 & 1100 & 10 & 8.2 & 1.8 & {$[12]$} \\
$06 / 28 / 00$ & 2.32 & 1400 & 2 & 8.1 & - & {$[3]$} \\
$07 / 03 / 02$ & 1.63 & 1700 & 5 & 8.0 & 2.2 & {$[13]$} \\
$22 / 03 / 02$ & 4.30 & 1460 & 0.011 & 7.3 & 2.1 & {$[2]$} \\
$07 / 05 / 04$ & 1.86 & 690 & 5 & $<7.0$ & - & {$[15]$} \\
\hline
\end{tabular}

TABLE 2. Physical Parameter at a CME-driven Shock derived by Bemporad \& Mancuso (2010)

\begin{tabular}{ccccc}
\hline & $\mathbf{T}_{e}\left(10^{6} \mathbf{K}\right)$ & $\mathbf{n}_{e}\left(\mathbf{1 0}^{-4} \mathbf{c m}^{-3}\right)$ & $\mathbf{v}\left(\mathbf{k m ~ s}^{-1}\right)$ & $\mathbf{B}(\mathbf{m G})$ \\
\hline upstream & 0.23 & 1.1 & 100 & 19 \\
downstream & 1.9 & 2.3 & 424 & 37 \\
\hline
\end{tabular}

interplanetary shocks, the heating of ions tends to decrease for increasing values of $\beta$.

Nevertheless, the interpretation is not always straightforward because the line broadening may also be due to non-thermal effects from ions energized at the shock front [9] and to the expansion of the CME front. For instance, after studying 22 full and partialhalo CMEs observed by UVCS, [4] concluded that, by assuming hemispherical shapes for the shock fronts, the $\mathrm{O}$ VI $\lambda 1032$ line broadenings in 3 events can be entirely ascribed to the LOS component of the bulk expansion, while this explanation was inadequate in 5 events. A correct estimate of the post-shock kinetic temperatures requires a subtraction of the line broadening due to the plasma expansion along the LOS.

There is a high degree of complementarity between the coronagraphic (spatial distribution, speed of driver and shock) and spectroscopic measurements (pre- and post-shock plasma parameters). Indeed, combined analyses of LASCO and UVCS observations can obtain the full range of plasma parameters upstream and downstream of the shock, including the magnetic field in both regimes. The MHD Rankine-Hugoniot jump relations for an oblique shock provide not only the post-shock plasma parameters, but also the pre- and post-shock magnetic field strength when the pre-shock coronal plasma parameters, with the exception of the magnetic field, can be determined by spectroscopic measurements (i.e., electron density and temperature, pre-shock and shock velocity) and the density compression ratio across the shock is determined by coronagraphic measurements. Such results derived by [2] are summarized in Table 2. Interestingly, the magnetic and kinetic energy density increases are nearly equal (as expected for equipartition of energy), and are both more than two times larger than the thermal energy increase. This trailblazing study demonstrates the power behing off-limb spectroscopic observations when combined with coronagraphic CME images. 


\section{CONCLUSIONS}

In the short space of this article, we tried to demonstrate the ability of coronagraphic and spectroscopic instruments to remotely detect coronal shocks and measure their properties. Despite a slow start during the 2000s, shocks are now detected and analyzed with increasing frequency.

Coronagraphs, especially the simultaneous observations from SECCHI and LASCO, are capable of deriving the 3D structure of the shock, its standoff distance from the driver, its speed and even the density compression ratio across the shock boundary. But they do not measure magnetic field or temperature conditions. Hence, coronagraphs cannot directly discriminate between a shock or a fast mode wave since the observed density compression could be the same. However, shocks can now be followed to at least 0.5 AU and possibly to Earth thanks to recent imag processing developments [5]. More details can be found in [25].

The spectroscopic observations have both advantages and disadvantages compared to the obsevations in the radio or in the visible. On one hand, the narrow slits provide only one-dimensional spatial information and the results are quite sensitive to the orientation of the slit relative to the shock front. Measurements of very fast shocks may also be compromised due to the relatively long integrations for the UVCS spectra $(\sim 100 \mathrm{~s})$. Spectroscopic detection of coronal shocks occurs only by chance, because the field of view of the $40^{\prime}$ long UVCS slit covers only a limited latitudinal region (e.g. $75^{\circ}$ when the slit is positioned at $1.7 \mathrm{R}_{\odot}$ ). On the other hand, spectral measurements are the only means to study the heating of individual ion species and electrons in the collisionless coronal shocks. Measurement of physical properties in CME-driven shocks and their correlations with SEP events will be very useful in establishing the necessary conditions for shocks to accelerate ambient solar wind or suprathermal ions to SEPs. Fortunately, off-limb spectroscopic capability will be offered by the forthcoming Solar Orbiter mission with the METIS [Multi-Element Telescope for Imaging and Spectroscopy; see 1] instrument, that will acquire simultaneously off-limb spectra at three heights, thus helping us to disentangle spatial and temporal evolution of plasma parameters across shocks.

\section{ACKNOWLEDGMENTS}

Part of this work was funded by various NASA grants. SOHO is an international collaboration between NASA and ESA. LASCO was constructed by a consortium of institutions: NRL (Washington, DC, USA), MPS (Katlenburg- Lindau, Germany), LAM (Marseille, France) and Univ.of Birmingham (Birmingham, UK). The SECCHI data are produced by an international consortium of the NRL, LMSAL and NASA GSFC (USA), RAL and Univ. Bham (UK), MPS (Germany), CSL (Belgium), IOTA and IAS (France).

\section{REFERENCES}

1. E. Antonucci, Mem. S.A.It., 82, 412-419 (2011)

2. A. Bemporad, \& S. Mancuso, Astroph. J., 720, 130-143 (2010) 
3. A. Ciaravella, et al., Astroph. J., 621, 1121-1128 (2005)

4. A. Ciaravella, et al., Astroph. J., 652, 774-792 (2006)

5. C. DeForest et al., arXiv:1104.1615 (2011)

6. N. Gopalswamy, et al., J. Geoph. Res., 110, A9, A09S15 (2005)

7. N. Gopalswamy, et al., Sol. Phys., 259, 227-254 (2009)

8. N. Gopalswamy \& S. Yashiro, Astrophys. J., 736, L17 (2011)

9. S. W. Kahler, et al., Solar Wind Nine AIP Conf. Proc., 471, 685-688 (1999)

10. K. E. Korreck, et al., Astroph. J., 659, 773-779 (2007)

11. L. C. Lee, et al., Geophys. Res. Lett., 13, 209 (1986)

12. S. Mancuso, S., et al., Astron. Astrophys., 383, 267-274 (2002)

13. S. Mancuso, \& D. Avetta, Astroph. J., 677, 683-691 (2008)

14. S. Mancuso, \& A. Bemporad, Adv. Sp. Res., 44, 451-456 (2009)

15. S. Mancuso, Sol. Phys., 96 (2011)

16. S. A. Maloney,\& P. T. Gallagher, arXiv:1106.1593 (2011)

17. G. Noci, Astrophy. J, 315, 706-715 (1987)

18. V. Ontiveros, \& A. Vourlidas, Astrophy. J, 693, 267-275 (2009)

19. N.-E. Raouafi, et al., Astron. Astrophys., 424, 1039-1048 (2004)

20. J. C. Raymond, et al. Geoph. J. Lett., 27, 1439-1442 (2000)

21. A. P. Rouillard, et al. Astroph. J., 735, 7-18 (2011)

22. M. Temmer, et al., Sol. Phys., 75 (2011)

23. A. Thernisien, A. Vourlidas, \& R. A. Howard, Sol. Phys.,256, 111-130 (2009)

24. A. Vourlidas, et al, Astroph. J., 598, 1392-1402 (2003)

25. A. Vourlidas, \& V. Ontiveros, "A Review of Coronagraphic Observations of Shocks Driven by Coronal Mass Ejections", in Shock Waves in Space and Astrophysical Environments, AIP Conference Proceedings, 1183, 2009, pp. 139-146 